\title{
Primary intraosseous squamous cell carcinoma of the mandible arising from an infected odontogenic cyst: A case report and review of the literature
}

\author{
KOENRAAD GRISAR ${ }^{1}$, MATTHIAS SCHOL ${ }^{2}$, ESTHER HAUBEN ${ }^{3}$, \\ JOSEPH SCHOENAERS $^{1}$ and CONSTANTINUS POLITIS ${ }^{1}$ \\ Departments of ${ }^{1}$ Oral and Maxillofacial Surgery, ${ }^{2}$ Dentistry and ${ }^{3}$ Imaging and Pathology, \\ University Hospitals Leuven, Leuven, 3000 Flemish Brabant, Belgium \\ Received October 31, 2015; Accepted May 19, 2016
}

DOI: $10.3892 / \mathrm{ol} .2016 .5378$

\begin{abstract}
Primary intraosseous squamous cell carcinoma (PIOSCC) derived from a odontogenic cyst is a rare form of odontogenic carcinoma. The incidence of carcinomas arising from odontogenic cysts is particularly uncommon and is reported to occur in 1-2 individuals for every 1,000 cases. The present case describes a 25-year-old man who was initially diagnosed with a chronically infected odontogenic cyst of the mandible. Biopsy and subsequent histology revealed the presence of squamous cell carcinoma. Therefore, neck dissection and hemimandibulectomy were performed. Ultimately, the situation in the mouth healed, though with a severe amount of scarring. Although the development of PIOSCC from an odontogenic cyst is rare, it should be included in the differential diagnosis for jaw bone radiolucency.
\end{abstract}

\section{Introduction}

Primary intraosseous squamous cell carcinoma (PIOSCC) is a rare lesion defined as an SCC that develops within the jaw bones, with no initial connection to the oral mucosa, and arises from remnants of odontogenic epithelium (1).

Loos (2) first defined PIOSCC in 1913 as 'central epidermoid carcinoma of the jaw', followed by Willis (3) in 1948 who described the lesion as an 'intra-alveolar epidermoid carcinoma'. This description was modified by Shear (4) to 'primary intra-alveolar epidermoid carcinoma' in 1969. In 1971, the term 'primary intraosseous carcinoma' (PIOC) was introduced (5). Elzay (6) reviewed the relevant literature associated with PIOC of the jaw and suggested an alteration to the

Correspondence to: Dr Koenraad Grisar, Department of Oral and Maxillofacial Surgery, University Hospitals Leuven, Kapucijnenvoer 33, Leuven, 3000 Flemish Brabant, Belgium E-mail: koenraadgrisar@gmail.com

Key words: primary intraosseous squamous cell carcinoma, odontogenic cyst
World Health Organization (WHO) classification to include 'ameloblastic carcinoma', which was later modified to account for potential etiological factors (7). Intraosseous mucoepidermoid carcinoma was also later included as an additional form of PIOC (8).

According to the 2005 WHO classification of tumors, PIOSCC is subcategorized into three different types: i) PIOSCC solid type (de novo); ii) PIOSCC originating from keratocystic odontogenic tumors; and iiiß§) PIOSCC originating from odontogenic cysts (9).

PIOSCC is estimated to account for $1-2 \%$ of all cases of oral cancer (1). The majority of PIOSCCs arise from odontogenic cysts, including dentigerous cysts, residual periapical cysts and keratocystic odontogenic tumors, which were previously known as odontogenic keratocysts $(1,10)$.

Commonly reported clinical features of PIOSCC include jaw swelling, pain and sensory disturbances $(1,11)$. Prior to the diagnosis of PIOSCC, the existence of a primary tumor in another site must be ruled out (11). The histological diagnosis is complicated by the fact that the histological features of PIOSCC are not pathognomonic (11).

With regard to patient survival, in 116 PIOSCC cases, Bodner et al found the overall 2- and 5-year survival rates to be 62 and $38 \%$, respectively (1).

The present study describes the case of a PIOSCC that originated from an ex-infected odontogenic cyst in the mandible, in addition to a review of the relevant literature.

\section{Case report}

In January 2013, a 25-year-old male patient presented to the Department of Oral and Maxillofacial Surgery, University Hospitals of Leuven (Leuven, Belgium). In November 2011, the patient was diagnosed with a benign odontogenic cyst of the right mandible, which was supported by panoramic radiography and computed tomography (CT) (Figs. 1 and 2). However, following initial marsupialization, the cyst recurred and a second surgery was performed in May 2012, which consisted of enucleation and reconstruction of the bony defect using an iliac crest graft. Nevertheless, the patient began to notice recurrence of the swelling, and 6 months later, a further 
A

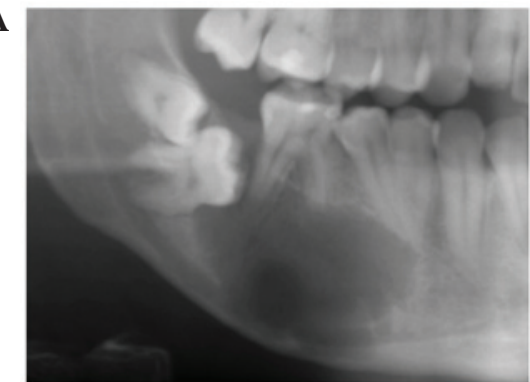

$\mathbf{B}$

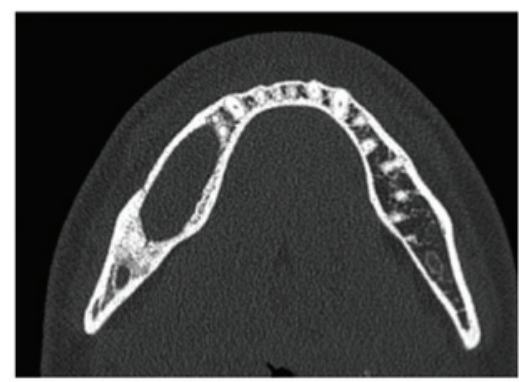

Figure 1. Cropped panoramic radiography and coronal CT images taken in November 2011 showing a unilocular radiolucent lesion in the right mandible. (A) Radiograph showing external root resorption of the distal root and extensive bony lysis over the apex of the first molar from the follicular space of the impacted second molar. (B) CT scan showing expansion of the buccal and lingual cortical plates. CT, computed tomography.

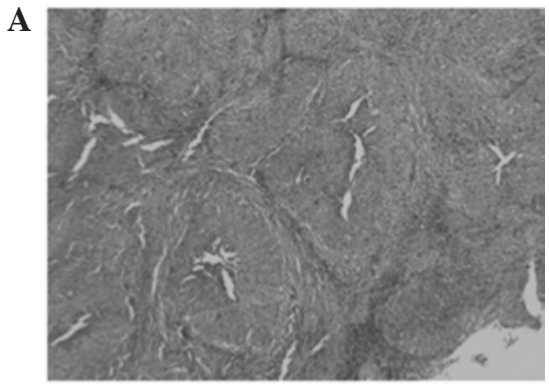

B

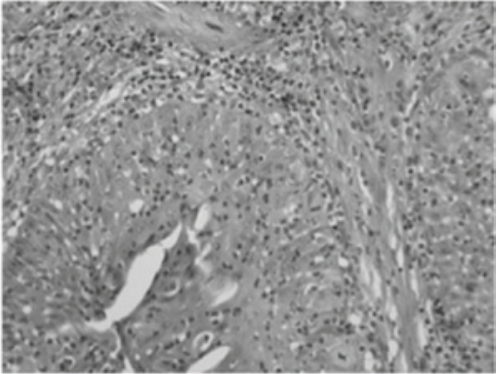

Figure 2. Histopathological examination of resected specimen. (A) Tumor tissue fragments are composed of a cell-rich, fibrous stroma in which a dense, mixed cellular infiltrate of lymphocytes, plasma cells and neutrophils are located. The fragments are lined with a multilayered squamous epithelium of varying width, with irregularly elongated epithelial ridges. The epithelium is partially folded in a papillomatous pattern, and the fragments exhibit deep epithelial invaginations. (B) The epithelium is infiltrated by neutrophil granulocytes, and multinucleated giant cells are present locally in the epithelium and just below the epithelial layer. Palisading of the basal cell layer is not observed. In certain areas, foci with slight parakeratosis are present. Staining, hematoxylin and eosin; magnification, $\mathrm{x} 40$.

surgical intervention was required. No improvement was noted, and the patient was subsequently referred to the Department of Oral and Maxillofacial Surgery, University Hospitals of Leuven.
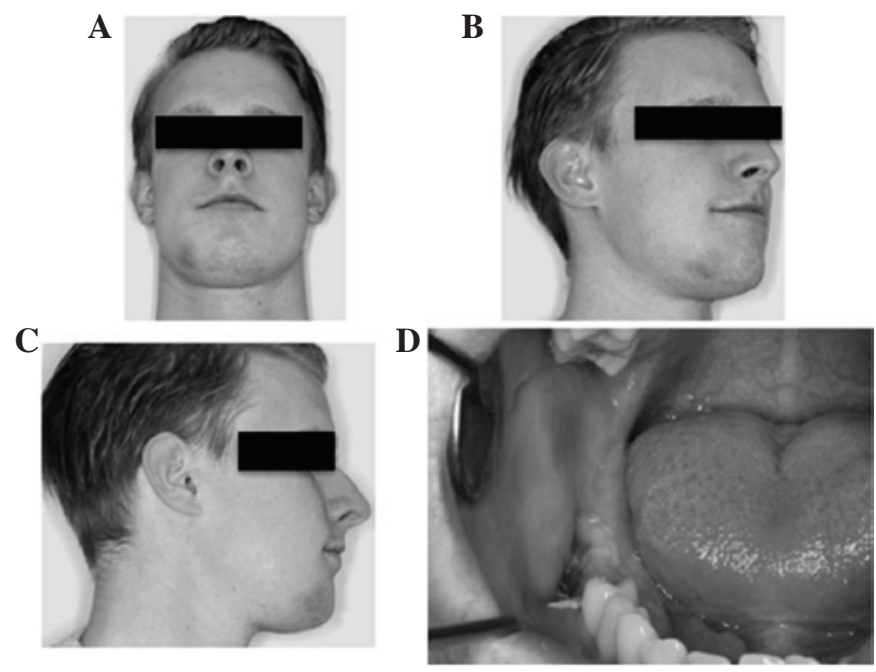

Figure 3. Images from initial examination in January 2013. (A-C) Buccal asymmetry was observed on the right side of the patient's face. (D) Intraoral images were captured showing obliteration of the right buccal sulcus.

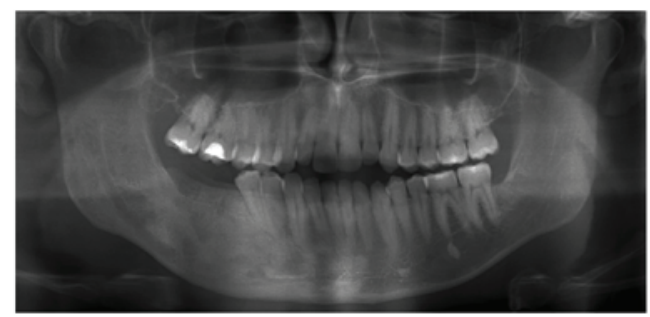

Figure 4. A panoramic radiograph taken in January 2013 reveals irregular areas of radiolucency along the right mandible in the molar region.

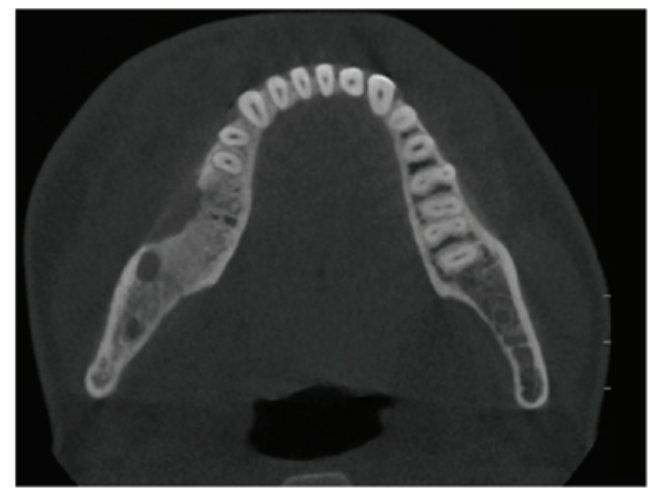

Figure 5. Cone beam computed tomography performed in January 2013 exhibits an extensive and poorly-defined bony destruction with perforation of the buccal and lingual cortical plates.

Initial observations revealed a right buccal swelling, a paste-like aspect to the skin and hypoesthesia of the right mental nerve. Apart from this, the patient reported no other sensory disturbances. Sensibility in the area innervated by the lingual nerve was intact. An intraoral examination identified swelling in the vestibular region and discharge along the right mandible in the molar region (Fig. 3). Given the clinical symptoms of inflammation, an initial diagnosis of osteomyelitis of the mandible was made. 


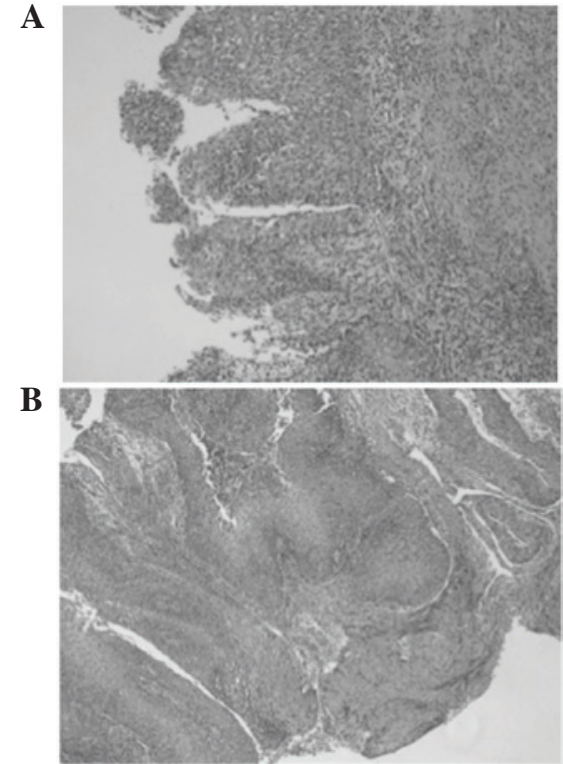

Figure 6. Histopathological examination of the resected specimen in May 2012. (A) Tumor tissue fragments are lined by a multilayered squamous epithelium without atypia, although the epithelial lining is prominent with infolding into the underlying stroma (magnification, x100). (B) Osteomyelitis was clinically present, and the image shows that the epithelium is heavily infiltrated by neutrophils. Staining, hematoxylin and eosin; magnification, x50).

The additional medical history of the patient was insignificant, while the dental history noted the extraction of four third molars and the lower right second molar 2 years previously. The patient's family and personal history was non-contributory. It was noted that the individual was allergic to penicillin.

Laboratory results, including routine blood work with checking of infectious parameters and a CBC, revealed no abnormalities. A panoramic radiograph detected irregular regions of radiolucency along the right mandible in the molar region (Fig. 4). A cone beam CT scan supported the clinical diagnosis of osteomyelitis of the mandible (Fig. 5). Wound debridement and buccal decortication were performed.

Histology of the resected specimen identified tissue fragments lined with a multilayered squamous epithelium with focal parakeratosis. The epithelium was densely infiltrated by neutrophils (Fig. 6). No evidence of malignancy was observed. Tissue cultures demonstrated the growth of Actinomyces species. An infectious disease physician (Department of Laboratory Medicine, University Hospitals of Leuven) was consulted and the patient was administered intravenous antibiotics, consisting of vancomycin $(1,000 \mathrm{mg} 2$ times per day for 7 days) and levofloxacin (500 $\mathrm{mg}$ for 7 days). In addition, adjunctive therapy consisting of 20 sessions of hyperbaric oxygen over an interval of 4 weeks was initiated.

Despite multiple debridement procedures, frequent follow-up and the administration of intravenous antibiotics, the symptoms did not resolve. Repeated biopsies consistently demonstrated no evidence of malignancy; however, it is possible that the well-differentiated nature of the lesion, with little or no atypia, and the background of long-term chronic inflammation with repeated successive interventions disturbing the histological picture prevented an early diagnosis of SCC.

In May 2013, 1 year after initial resection of the recurrent lesion and reconstruction with an iliac crest bone graft, a new

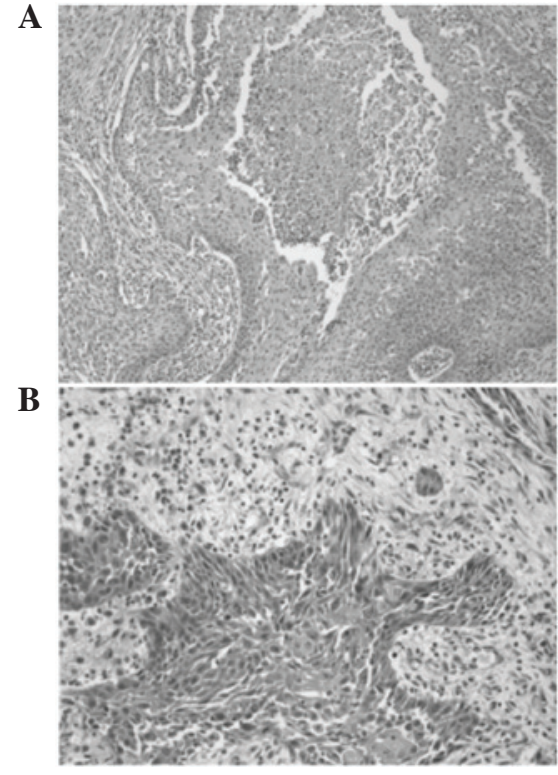

Figure 7. Histopathological examination of the sixth lesion recurrence in May 2013. (A) The epithelial nests pushing into the stroma are more prominent compared with previous histopathology. Central necrosis is present in the nests and atypia is minimal (magnification, x100). (B) The normal layering and maturation is disturbed, nuclear atypia is observed and small epithelial strands infiltrating the surrounding stroma are present (magnification, x200). Staining, hematoxylin and eosin.

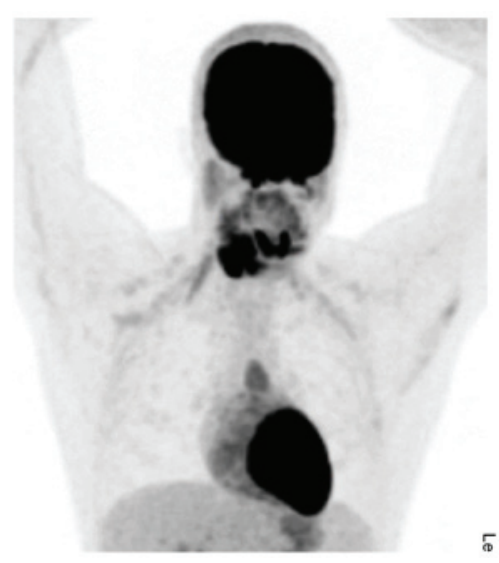

Figure 8. FDG positron emission tomography/computed tomography revealed FDG accumulation in the lesion on the right mandible and a limited hypermetabolic mass in the anterior mediastinum. FDG, ${ }^{18} \mathrm{~F}$-fluorodeoxyglucose.

biopsy of the lesion was taken and histopathological examination of the specimen identified central necrosis, nuclear atypia and small epithelial strands infiltrating the surrounding stroma (Fig. 7).

Following combination of the radiological, histopathological and histochemical findings, the final diagnosis was confirmed as a PIOSCC, which had developed from an ex-infected odontogenic cyst. In addition to the known lesion, positron emission tomography revealed a limited hypermetabolic mass in the anterior mediastinum (Fig. 8).

The case was categorized as $\mathrm{pT} 4 \mathrm{pNO}$ and a multidisciplinary decision was made for the patient to undergo complete surgical excision of the lesion, followed by postoperative radiotherapy (60 Gy in 30 sessions of 2 Gy each). 


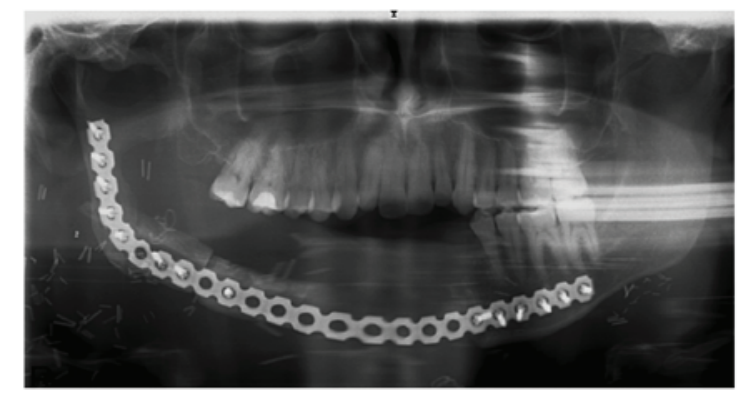

Figure 9. Panoramic radiograph following right hemimandibulectomy and neck dissection. Reconstruction was subsequently performed with an osteomyocutaneous fibular flap.
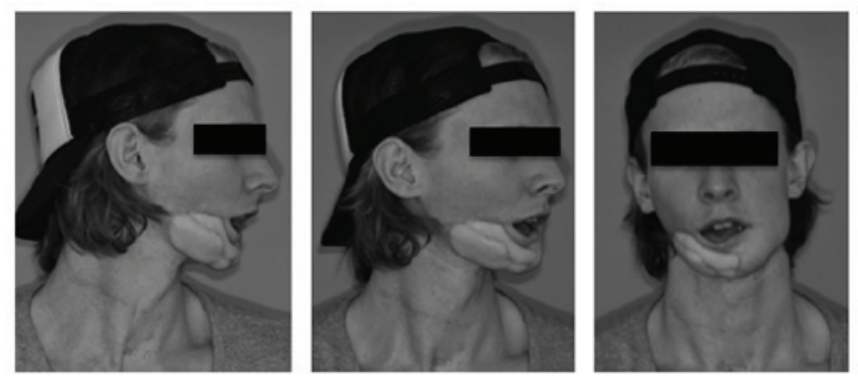

Figure 10. Clinical images taken in September 2014 subsequent to right hemimandibulectomy, neck dissection and reconstruction with an osteomyocutaneous fibular flap.

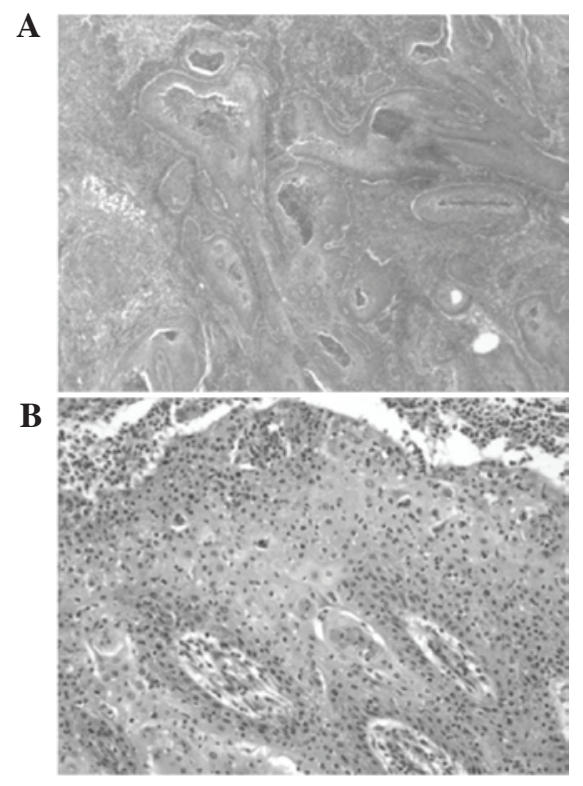

Figure 11. Histopathological examination of the final resected specimen (A) Low power images showing a large, infiltrative nest of squamous epithelium with central necrosis (magnification, x25). (B) The tumor cells are moderately atypical, and mitotic and dyskeratotic cells are present (magnification, x200).Staining, hematoxylin and eosin.

Genetic sequencing did not identify any mutations in the patched 1 gene; therefore, the presence of basal cell nevus syndrome was highly improbable.

In June 2013, a right hemimandibulectomy was performed from the left canine region to the right subcondylar region, in addition to a supraomohyoid right neck dissection. Resection margins were all clear, and no tumor was observed in the lymph nodes. Reconstruction with an osteomyocutaneous fibular flap was performed (Figs. 9 and 10).

During the postoperative final lesion biopsy, a welldifferentiated SCC was confirmed, with a $7.5-\mathrm{cm}$ diameter, expansive growth without lymphatic or perineural invasion, and free surgical margins. No metastasis was observed in the level I, II and III lymph nodes (Fig. 11).

The reconstruction with a free, vascularized, fibular graft, for reasons not well understood, became infected and after several phases of surgical debridement, it was decided that a new reconstruction would be performed with a second free fibular graft from the other leg. Ultimately, the situation in the mouth healed, though with a severe amount of scarring (Fig. 10). All biopsies subsequent to the first resection remained negative for malignancy recurrence.

At 18 months post-resection of the SCC, a hypermetabolic mass in the anterior mediastinum was reported, which was diagnosed as T-cell acute lymphoblastic leukemia and treated accordingly with chemotherapy consisting of $1.2 \mathrm{~g} / \mathrm{m}^{2}$ cyclofosfamide (days 1 and 17), $1.4 \mathrm{mg} / \mathrm{m}^{2}$ vincristine (days 3,10 , 17, 24 and 31), $45 \mathrm{mg} / \mathrm{m}^{2}$ daunorubicine (days 3 and 4) and $10,000 \mathrm{U} / \mathrm{m}^{2}$ asparaginase (days 3, 5, 7, 9, 11, 13 and 15).

At the 2-year follow-up, the patient was without further evidence of recurrence. Due to the fact that the patient preferred to continue treatment in a hospital closer to his residence, there has not been follow-up of the patient in University Hospitals Leuven since November 2015.

\section{Discussion}

PIOSCC is a form of SCC that arises within the jaw bones from remnants of the odontogenic epithelium, without a connection to the oral mucosa (1). According to the 2005 WHO classification of tumors, PIOSCC is subcategorized into three different types: i) PIOSCC solid type (de novo); ii) PIOSCC derived from keratocystic odontogenic tumors; and iii) PIOSCC derived from odontogenic cysts (9). Bodner et al (1) reported that the majority of PIOSCC cases $(85 \%)$ are associated with moderately- or well-differentiated SCCs. In addition, the overall survival rate of patients with a PIOSCC at 2 and 5 years was 62 and $38 \%$, respectively (1).

Despite classification of the disease improving throughout the years, the etiology and pathogenesis remain to be fully understood, and the factors underlying the malignant transformation of the benign cystic lining of odontogenic cysts are not known (12). It has been suggested that chronic inflammation from infection of an odontogenic cyst may serve as a key factor in carcinogenesis (13-15). This is based on the observation that chronic infiltration of plasma cells and lymphocytes in the connective tissue of the cyst wall accompanied the malignant transformation of cyst epithelium (1).

The current case presented with a persistent infection that did not respond to treatment. It is likely that the discharge and recurrent infections were caused by the underlying malignancy. Since infected odontogenic cysts tend to respond to treatment with intravenous or oral antibiotics, persistent infections associated with odontogenic cysts should encourage the clinician to consider the possibility of an underlying malignancy (16). 
The possibility of chronic infection leading to tumor formation cannot be excluded.

Radiological and clinical characteristics of PIOSCCs are similar to those of benign odontogenic cysts and tumors. The most commonly reported symptoms in patients with PIOSCC are swelling and pain, and the mandible is more frequently involved $(79 \%)$ than the maxilla $(21 \%)(1,17)$. Sensory disturbances, including numbness and paresthesia, were also experienced in the present case. A sensory disturbance of the jaw, particularly one with no history of trauma, should always be regarded as a potential symptom of malignancy, including PIOSCC (18).

In certain cases, early-stage PIOSCC may mimic routine dental disorders, such as periapical and periodontal disease, which may lead to misdiagnosis or delayed diagnosis $(15,17)$. As malignant tumors that have metastasized to the jaw from distant sites, tumors originating from the maxillary sinus, and alveolar carcinomas that have invaded the bone from the surface must be ruled out, the definitive diagnosis of PIOSCC is often challenging (11).

The treatment of choice for PIOSCC is surgery and/or radiation therapy $(18,19)$. Surgery consists of en bloc resection of the lesion followed by reconstruction and, if indicated, neck dissection (16). Postoperatively, chemoradiotherapy may be more effective than radiotherapy alone (10).

Although genetic counseling in the present case identified no familial factors, the conversion to a malignant tumor and the subsequent emergence of a hematological tumor raise suspicions to the existence of a malignant predisposition of an unknown origin.

In conclusion, the current study described a case of PIOSCC of the mandible, which, based on its clinical course, most likely originated from an infected odontogenic cyst. Clinicians must be aware that PIOSCC may initially present as a routine dental disorder, and that unsuccessful treatment and recurrences may be a sign of underlying malignancy. Although occurrence of the hematological malignancy in the present case may have been coincidental, the occurrence may also be based on a shared genetic etiology.

\section{References}

1. Bodner L, Manor E, Shear M and van der Waal I: Primary intraosseous squamous cell carcinoma arising in an odontogenic cyst: A clinicopathologic analysis of 116 reported cases. J Oral Pathol Med 40: 733-738, 2011.
2. Loos D: Central epidermoid carcinoma of the jaws. Dtsch Monatsschr Zahnheilk 31: 308, 1913 (In German).

3. Willis RA: Pathology of Tumors. Mosby, St. Louis, pp310-316, 1948.

4. Shear M: Primary intra-alveolar epidermoid carcinoma of the jaw. J Pathol 97: 645-651, 1969.

5. Pindborg JJ, Kramer IRH and Torloni H (eds). International Histological Classification of Tumours No. 5: Histological Typing of Odontogenic Tumours, Jaw Cysts and Allied Lesions. World Health Organization, pp35-36, 1972.

6. Elzay RP: Primary intraosseous carcinoma of the jaws: Review and update of odontogenic carcinoma. Oral Surg Oral Med Oral Pathol 54: 299-303, 1982.

7. Slootweg PJ and Müller H: Malignant ameloblastoma or ameloblastic carcinoma. Oral Surg 57: 168-176, 1984.

8. Waldron CA and Mustoe TA: Primary intraosseous carcinoma of mandible with probable origin in an odontogenic cyst. Oral Surg Oral Med Oral Pathol 67: 716-724, 1989.

9. Eversole LR, Siar CH and van der Waal I: Primary intraosseous squamous cell carcinomas. In: World Health Organization Classification of Tumors. Pathology and Genetics Head and Neck Tumors. Barnes L, Evson JW, Reichart P and Sidransky D (eds). Lyon, IARC Press, pp290-291, 2005.

10. Woolgar JA, Triantafyllou A, Ferlito A, Devaney KO, Lewis JS Jr, Rinaldo A, Slootweg PJ and Barnes L: Intraosseous carcinoma of the jaws: A clinicopathologic review. Part III: Primary intraosseous squamous cell carcinoma. Head Neck 35: 906-909, 2013.

11. Suei Y, Tanimoto K, Taguchi A and Wada T: Primary intraosseous carcinoma: Review of the literature and diagnostic criteria. J Oral Maxillofac Surg 52: 580-583, 1994.

12. Jain M, Mittal S and Gupta DK: Primary intraosseous squamous cell carcinoma arising in odontogenic cysts: An insight in pathogenesis. J Oral Maxillofac Surg 71: e7-e14, 2013.

13. Gardner AF: The odontogenic cyst as a potential carcinoma: A clinicopathologic appraisal. J Am Dent Assoc 78: 746-755, 1969.

14. Coussens LM and Werb Z: Inflammation and cancer. Nature 420: 860-867, 2002.

15. Choi S and Myers JN: Molecular pathogenesis of oral squamous cell carcinoma: Implications for therapy. J Dent Res 87: 14-32, 2008.

16. Tan B, Yan TS, Shermin L, Teck KC, Yoke PC, Goh C and Balakrishnan A: Malignant transformation of keratocystic odontogenic tumor: Two case reports. Am J Otolaryngol 34: 357-361, 2013.

17. Müller S and Waldron CA: Primary intraosseous squamous carcinoma. Report of two cases. Int J Oral Maxillofac Surg 20: 362-365, 1991 .

18. Thomas G, Pandey M, Mathew A, Abraham EK, Francis A, Somanathan T, Iype M, Sebastian P and Nair MK: Primary intraosseous carcinoma of the jaw: Pooled analysis of world literature and report of two new cases. Int J Oral Maxillofac Surg 30: 349-355, 2001.

19. Zwetyenga N, Pinsolle J, Rivel J, Majoufre-Lefebvre C, Faucher A and Pinsolle V: Primary intraosseous carcinoma of the jaws. Arch Otolaryngol Head Neck Surg 127: 794-797, 2001. 